\section{REVISTA BRASILEIRA DE QUALIDADE DE VIDA}

\title{
Avaliação da qualidade de vida, nível de Burnout e enfrentamento do estresse no trabalho de agentes comunitários de uma unidade de Programa de Saúde da Família no município de Piracicaba/SP
}

\author{
Evaluation of the quality of life, level of Burnout and coping occupational of \\ community health agents of a unity of Family Health Program, in Piracicaba/SP
}

\author{
Paigy Costa \\ Universidade Metodista de Piracicaba - UNIMEP - Piracicaba - São Paulo - Brasil \\ paigycosta@gmail.com \\ Silvia Belissa Ferrareto \\ Universidade Metodista de Piracicaba - UNIMEP - Piracicaba - São Paulo - Brasil \\ silviabelissa@hotmail.com \\ Gislaine Cecília de Oliveira Cerveny \\ Universidade Metodista de Piracicaba - UNIMEP - Piracicaba - São Paulo - Brasil \\ gicerveny@yahoo.com.br
}

\section{RESUMO}

OBJETIVO: Avaliar a qualidade de vida, o nível Burnout e as estratégias de enfrentamento do estresse de agentes comunitários de saúde (ACSs), e possível correlação entre as variáveis.

MÉTODOS: Estudo transversal descritivo. O estudo foi composto por seis ACSs de uma unidade do Programa de Saúde da Família e a avaliação feita através da aplicação de três questionários. Para avaliar a qualidade de vida, foi aplicado o Questionário de WHOQOL-bref e para o nível de Burnout, o questionário de Jibeli (2009). Para avaliar as estratégias de enfrentamento do estresse utilizadas pelos ACSs, foi aplicada a Escala de Coping Ocupacional. Por fim, foi realizada a correlação entre os quatro componentes da qualidade de vida, o nível de Burnout e os três fatores de enfrentamento do estresse, por meio do teste de correlação de Spearman $(\mathrm{p}<0,05)$.

RESULTADOS: Entre os resultados referentes à qualidade de vida dos agentes, o domínio social obteve o maior resultado entre os quatro componentes, seguido pelos domínios psicológico, físico e meio ambiente. A maioria dos ACSs apresentou um nível de Burnout considerável (quatro agentes com nível Burnout considerável e dois com Burnout em instalação). Entre as estratégias de enfretamento do estresse, o fator controle foi a mais utilizada, seguidos pelos fatores esquiva e manejo de sintomas. Entre as variáveis dos questionários, o fator esquiva (Coping ocupacional) e o domínio psicológico (Qualidade de vida) apresentaram correlação forte $(0,882 ; p=0,020)$, assim como o domínio social (Qualidade de vida) e o domínio psicológico (Qualidade de vida) (0,925; $\mathrm{p}=0,008)$ e o domínio físico (Qualidade de vida) com o domínio ambiente (Qualidade de vida) $(0,851 ; \mathrm{p}=0,032)$.

CONCLUSÃO: Não foi observada a correlação entre estresse e possíveis alterações na qualidade de vida e fatores de enfrentamento, mas foram encontradas influências entre técnicas de enfrentamento do estresse e alterações da qualidade de vida. 
PALAVRAS-CHAVE: Qualidade de vida. Estresse. Burnout.

\begin{abstract}
OBJECTIVE: Assess the quality of life, Burnout level and stress coping strategies of community health agents, and correlation between the variables.

METHODS: Cross-sectional study. This study was composed for 6 community health agents of a unity of Family Health Program. For the evaluation of quality of life of community health agents, the WHOQOL-bref questionnaire was applied. For asses the Burnout level, was chosen the Jibeli (2009) questionnaire. For the evaluation of stress coping strategies, the coping occupational scale was applied. The Spearman rank correlation coefficient among the domains of the inter and intra groups questionnaire was applied $(\mathrm{p}<0,05)$.

RESULTS: Among the results of the quality of life of agents, the social domain got the highest score among the four components, followed by psychological, physical and environmental domain. Most community health agents showed a considerable level of Burnout (four agents with considerable Burnout level and two with Burnout in installation). Between coping strategies of stress, the control factor was the strategy most used by the agents, followed by avoidance factor and finally the management of symptoms factor. Among the variables of the questionnaires, the factor avoidance (Occupational coping) and psychological domain (Quality of life) were strongly correlated $(0.0882, \mathrm{p}=0.020$, as well as the social domain (Quality of life) and the psychological domain (Quality of life) $(0.925, \mathrm{p}=0.008)$ and the physical domain (Quality of life) with the domain environment (Quality of life) $(0.851, \mathrm{p}=0.032)$.

CONCLUSIONS: The control factor, stress coping strategy, was used more often by the community health agents. Four, of the six community health agents, had the Burnout installed. The variables that were correlated: the elusive factor in the psychological domain, social domain with the psychological domain and the physical domain on the environment domain environment.
\end{abstract}

KEYWORDS: Quality of life. Stress. Burnout.

\title{
1 Introdução
}

A qualidade de vida é resultante de diversos fatores, não só individuais, mas também sociais. De acordo com Minayo, Hartz e Buss (2000), a qualidade de vida representa o grau de satisfação encontrado no relacionamento familiar, amoroso e social, além de contribuições ambientais.

O trabalho proporciona várias realizações pessoais e é um importante representante com influência direta e significativa na qualidade de vida do indivíduo. Entretanto, o trabalho pode influenciar de forma negativa a qualidade de vida, se os fatores de riscos à saúde, que estão relacionados a esta, superarem os instrumentos de proteção (MURTA; TRÓCCOLI, 2004).

$\mathrm{O}$ estresse é um destes riscos, e muitos estudos vêm sendo realizados para reportar o surgimento e os efeitos deste em trabalhadores de centros de saúde (CAMELO, 2006; LORENZ; BENATTI; SABINO, 2010).

De acordo com Lopes (2011), a taxa de auxílio doença para os trabalhadores acometidos pelo estresse teve aumento de mais de $28 \%$ no primeiro semestre de 2011 em relação ao mesmo período do ano de 2010 ( 85 mil casos em 2010 - 109 mil casos em 2011).

As consequências advindas do estresse podem ser catastróficas ao organismo do trabalhador, afetando os sistemas musculoesquelético e cardiovascular, a cognição e o sono (LENTINE; SONODA; BIAZIN, 2003; CAMELO, 2006; ROCHA; MARTINO, 2010; BARBIRATO et al., 2010). Porém, os sistemas cardiovascular e imunológico possuem respostas mais 'francas' em 
relação ao estresse. Como relatado por Loures et al. (2002), alterações como aumento da frequência cardíaca, da pressão arterial e da frequência respiratória, assim como a diminuição da resposta inflamatória/imune, entre outras, são encontradas em indivíduos quando o estresse já está instalado.

Distúrbios do sono, conforme observado por Rocha e Martino (2010), é outra consequência negativa que ocorre devido à instalação do estresse.

Os agentes comunitários de saúde (ACSs) fazem parte de uma equipe multiprofissional que aproxima a população dos serviços de saúde. Esta equipe faz parte do Programa de Saúde da Família (PSF) e é composta por pelo menos um médico, um enfermeiro, um auxiliar de enfermagem e de quatro a seis ACSs (SANTANA; CARMAGNANI, 2001). No entanto, os ACSs como quaisquer outros profissionais estão expostos a fatores de riscos que influenciam na instalação do estresse.

Os ACSs possuem funções específicas que regem sua importância na equipe multidisciplinar que compõe o PSF, também conhecido como Estratégias da Saúde da Família (ESF). De acordo com Vilela, Silva e Jackson Filho (2010), as atividades giram em torno da principal atribuição, as visitas domiciliares.

Os ACSs estão expostos à sobrecarga física e mental, salário abaixo do desejado, falta de estrutura física e material para o desenvolvimento da função, cobranças excessivas da população e dos supervisores, além da falta de apoio da equipe de saúde, sendo estas as principais variáveis que podem desenvolver ou atenuar o estresse nestes trabalhadores (SIMÕES, 2009).

O estresse em fase crônica é conhecido como Síndrome de Burnout, quadro comum em trabalhadores que tem contato direto e constante com a população (TELLES; PIMENTA, 2009). As principais consequências desta fase são: fadiga constante, falta de energia, distanciamento afetivo, insensibilidade, irritabilidade e insatisfação pessoal (VIEIRA, 2010).

Deste modo, o objetivo desse estudo foi avaliar a qualidade de vida, o nível Burnout e as estratégias de enfrentamento do estresse de ACSs, e possível correlação entre as variáveis.

\section{Material e métodos}

Trata-se de estudo do tipo descritivo transversal, de abordagem quantitativa, do qual participaram seis ACSs de uma unidade do PSF do município de Piracicaba/SP. O estudo foi aprovado pelo Comitê de Ética em Pesquisa (CEP) da Universidade Metodista de Piracicaba (UNIMEP), sob o protocolo $\mathrm{n}^{\circ}$ 92/11.

Foi realizada uma reunião mediante anuência dos ACSs e da chefia, com intuito de apresentar o objetivo do estudo, esclarecendo qualquer dúvida sobre os procedimentos e a participação dos agentes. Em seguida, os que optaram por participar como voluntários assinaram o termo de consentimento livre esclarecido, com base nas orientações da Resolução 196/96 do Conselho Nacional de Saúde.

Foram incluídos no estudo agentes que assinaram o termo de consentimento e que estivessem trabalhando desde a aplicação do primeiro até o último questionário (de abril a junho de 2012). Foram excluídos do estudo os agentes comunitários que estivessem em férias no período da aplicação dos três questionários.

Primeiramente foram coletados dados sobre a idade, grau de instrução e tempo na função dos ACSs. Os questionários foram aplicados pelo pesquisador, em data agendada com cada voluntário, sendo os mesmos identificados por meio de um código criado na entrada do estudo, para garantia da confidencialidade.

Para avaliar a qualidade de vida foi utilizado o questionário WHOQOL-bref da Organização Mundial da Saúde (OMS) (FLECK et al., 2000), para o qual os ACSs basearam suas respostas levando em consideração os últimos 15 dias. É um questionário composto por 26 questões, sendo que as 2 primeiras avaliam a qualidade de vida e satisfação da saúde geral. As 24 questões restantes são divididas em quatro domínios: físico (1, 2, 3, 9, 10, 11 e 12), psicológico (4, 5, 6, 7, 8 e 24), relações sociais $(13,14$ e 15$)$ e meio ambiente $(16,17,18,19,20,21,22$ e 23$)$. Foi realizada a 
sintaxe de acordo com Pedroso et al. (2010), das respostas dadas pelos ACSs, no qual o pior e o melhor resultado considerados, respectivamente, são 0 (ruim) e 100 (bom). De acordo com os achados de Saupe et al. (2004), os dados são avaliados de acordo com os seguintes parâmetros: 040 estão na 'região de fracasso', 41-71 é considerada 'região de indefiniç̧ão' e dados acima de 71 estão inseridos na 'região de sucesso'.

O nível de Burnout dos trabalhadores foi avaliado utilizando o questionário de Jibeli (2009), para identificação preliminar, o qual é inspirado no Maslach Burnout Inventory (MBI) (MASLACH; JACKSON, 1981). Este questionário é composto por 20 itens com respostas que variam entre 'nunca' (1) e 'diariamente' (5), e os trabalhadores se baseiam na quantidade de vezes que se sentiram nas situações apresentadas e sugeridas pelo questionário. Neste questionário as colunas de respostas $(1,2,3,4$ e 5) tiveram seus respectivos resultados somados entre si e o resultado final foi avaliado em forma de pontos. A avaliação do nível de Burnout foi identificada de acordo com o resultado da pontuação: 0-20 pontos (nenhum indício), 21-40 (possibilidade de desenvolver), 41-60 (fase inicial), 61-80 (início da instalação) e 81-100 (fase considerável).

O último questionário aplicado foi a Escala de Coping Ocupacional, utilizado para avaliar as ações de enfrentamento dos trabalhadores em relação ao estresse. Esta escala, formulada inicialmente por Latack (1986), foi traduzida e adaptada para estudos brasileiros por Pinheiro, Tróccoli e Tamayo (2003). A escala é composta por 29 itens, relacionados com a maneira que o trabalhador age de acordo com os problemas no ambiente de trabalho. Os autores dividiram os itens em três grupos de fatores: controle, esquiva e manejo de sintomas. Os grupos foram distribuídos da seguinte maneira no questionário: 9 itens relacionados ao fator esquiva, 11 itens ao fator controle e 9 itens ao fator manejo de sintomas. Tomando como base o estudo de Umann (2011), as respostas foram divididas em cinco opções, que variam desde 'nunca faço isso' (1) até 'sempre faço isso' (5). Foi calculada a média dos três grupos de fatores de cada trabalhador, para identificar o prevalente.

Todos os questionários utilizados foram aplicados uma única vez para cada agente comunitário.

Os dados foram normalizados e em seguida foram submetidos ao coeficiente de Spearman para verificar a existência de correlação entre os resultados dos questionários. A seguinte classificação foi adotada para os valores de correlação: correlação fraca <0,30; correlação moderada $\leq 0,70$ e correlação excelente $>0,70$.

Em todos os casos, os níveis de significância foram pré-fixados em 5\% (p<0,05).

\section{Resultados}

Os dados sobre a idade, grau de instrução e tempo de função de cada voluntário estão apresentados na Tabela 1:

Tabela 1 - Idade, grau de instrução e tempo como ACS

\begin{tabular}{lccc}
\hline & Idade (anos) & Grau de instrução & Tempo como ACS (anos) \\
\hline ACS 1 & 38 & Ensino fundamental & 24 meses \\
ACS 2 & 32 & Ensino médio & 132 meses \\
ACS 3 & 25 & Ensino médio incompleto & 72 meses \\
ACS 4 & 33 & Cursando o ensino superior & 114 meses \\
ACS 5 & 37 & Cursando o ensino superior & 35 meses \\
ACS 6 & 29 & Ensino superior incompleto & 36 meses \\
\hline Média & 32,3 & & 68,8 meses \\
Desvio padrão & $32,3 \pm 4,9$ anos & $68,8 \pm 45,3$ meses \\
\hline
\end{tabular}

Fonte: Autoria própria (2014).

A qualidade de vida foi a primeira variável analisada nos ACSs e os resultados estão expressos na Tabela 2: 
Tabela 2 - Qualidade de vida dos ACSs de acordo com a pontuação nos domínios físico, psicológico, social e meio ambiente

\begin{tabular}{lc}
\hline Domínios & Pontuação \\
\hline Físico & 55,4 \\
Psicológico & 58,3 \\
Social & 63,9 \\
Meio ambiente & 50,5 \\
\hline \multicolumn{2}{l}{ Fonte: Autoria própria (2014). }
\end{tabular}
da média.

No geral, os domínios do questionário de qualidade de vida apresentaram resultados acima

Também foram tabulados os resultados relativos ao nível de Burnout dos ACSs (Tabela 3).

Tabela 3 - Nível de Burnout dos ACSs, com pontuação e classificação correspondente para cada voluntário

\begin{tabular}{lcccccc} 
Código & ACS1 & ACS2 & ACS3 & ACS4 & ACS5 & ACS6 \\
\hline Pontuação & 81 & 72 & 79 & 95 & 82 & 94 \\
Resultado & $\begin{array}{c}\text { Burnout } \\
\text { considerável }\end{array}$ & $\begin{array}{c}\text { Burnout em } \\
\text { instalação }\end{array}$ & $\begin{array}{c}\text { Burnout em } \\
\text { instalação }\end{array}$ & $\begin{array}{c}\text { Burnout } \\
\text { considerável }\end{array}$ & $\begin{array}{c}\text { Burnout } \\
\text { considerável }\end{array}$ & $\begin{array}{c}\text { Burnout } \\
\text { considerável }\end{array}$ \\
\hline
\end{tabular}

Fonte: Autoria própria (2014).

Mais da metade dos agentes $(66,7 \%)$ apresentaram nível de Burnout considerável, enquanto no restante da amostra $(33,3 \%)$ o quadro encontra-se em instalação.

A seguir será mostrada a análise das estratégias de enfrentamento do estresse utilizada pelos ACSs (Tabela 4):

Tabela 4 - Estratégias de enfrentamento do estresse dos ACS, segundo o questionário de Coping Ocupacional, subcategorizadas nos fatores controle, esquiva e manejo de sintomas

\begin{tabular}{lccc}
\hline & Fator controle & Fator esquiva & Fator manejo de sintomas \\
\hline Média & 3,9 & 3,4 & 2,4 \\
Máximo & 5,0 & 5,0 & 5,0 \\
Mínimo & 1,0 & 1,0 & 1,0 \\
Desvio padrão & 0,9 & 1,3 & 1,4 \\
\hline \multicolumn{4}{c}{ Fonte: Autoria própria (2014). }
\end{tabular}

O fator controle das estratégias de enfrentamento do estresse foi o que obteve a maior média $(3,9)$ em relação às respostas utilizadas pelos agentes comunitárias e com maior prevalência entre elas $(66,7 \%)$.

A Tabela 5 apresenta os resultados de correlação entre as variáveis analisadas (estresse, formas de enfrentamento do estresse e setores da qualidade de vida). 
Tabela 5 - Valores do Coeficiente de Correlação de Spearman (CCS) das variáveis analisadas

\begin{tabular}{|c|c|c|c|c|c|c|c|c|c|}
\hline & & Esquiva & Controle & Manejo & Social & Físico & Psicológico & Ambiente & Burnout \\
\hline \multirow{2}{*}{ Esquiva } & CCS & 1,000 & 0,279 & 0,191 & 0,776 & 0,735 & 0,882 & 0,582 & $-0,493$ \\
\hline & $\mathrm{p}$ & . & 0,592 & 0,717 & 0,070 & 0,096 & $\underline{\mathbf{0 , 0 2 0}}$ & 0,225 & 0,321 \\
\hline \multirow{2}{*}{ Controle } & CCS & 0,279 & 1,000 & 0,353 & 0,403 & 0,015 & 0,500 & $-0,269$ & $-0,406$ \\
\hline & $\mathrm{p}$ & 0,592 & . & 0,493 & 0,428 & 0,978 & 0,313 & 0,607 & 0,425 \\
\hline \multirow{2}{*}{ Manejo } & $\mathrm{CCS}$ & 0,191 & 0,353 & 1,000 & 0,672 & 0,000 & 0,588 & $-0,403$ & $-0,319$ \\
\hline & $\mathrm{p}$ & 0,717 & 0,493 & . & 0,144 & 1,000 & 0,219 & 0,428 & 0,538 \\
\hline \multirow{2}{*}{$\underline{\text { Social }}$} & CCS & 0,776 & 0,403 & 0,672 & 1,000 & 0,313 & 0,925 & 0,091 & $-0,265$ \\
\hline & $\mathrm{p}$ & 0,070 & 0,428 & 0,144 & . & 0,545 & $\underline{0,008}$ & 0,864 & 0,612 \\
\hline \multirow[b]{2}{*}{ Físico } & CCS & 0,735 & 0,015 & 0,000 & 0,313 & 1,000 & 0,603 & 0,851 & $-0,551$ \\
\hline & $\mathrm{p}$ & 0,096 & 0,978 & 1,000 & 0,545 & . & 0,205 & $\underline{0,032}$ & 0,257 \\
\hline \multirow{3}{*}{ Psicológico } & CCS & 0,882 & 0,500 & 0,588 & 0,925 & 0,603 & 1,000 & 0,313 & $-0,493$ \\
\hline & $\mathrm{p}$ & $\underline{\mathbf{0 , 0 2 0}}$ & 0,313 & 0,219 & $\underline{0,008}$ & 0,205 & . & 0,545 & 0,321 \\
\hline & CCS & 0,582 & $-0,269$ & $-0,403$ & 0,091 & 0,851 & 0,313 & 1,000 & $-0,088$ \\
\hline \multirow[t]{2}{*}{ Ambiente } & $\mathrm{p}$ & 0,225 & 0,607 & 0,428 & 0,864 & $\underline{\mathbf{0 , 0 3 2}}$ & 0,545 & . & 0,868 \\
\hline & CCS & $-0,493$ & $-0,406$ & $-0,319$ & $-0,265$ & $-0,551$ & $-0,493$ & $-0,088$ & 1,000 \\
\hline Burnout & $\mathrm{p}$ & 0,321 & 0,425 & 0,538 & 0,612 & 0,257 & 0,321 & 0,868 & . \\
\hline
\end{tabular}

Fonte: Autoria própria (2014).

\section{Discussão}

De acordo com Seidl e Zannon (2004), o intuito da avaliação da qualidade de vida é indicar a relação entre a presença de enfermidades ou necessidades de intervenções e a saúde dos trabalhadores avaliados.

O domínio social dos ACSs neste estudo obteve 63,9, o qual se encontra na 'região de indefinição', sendo um resultado insuficiente para uma boa qualidade de vida. Este valor se aproxima dos dados de Paschoa, Zanei e Whitaker (2007), que avaliaram enfermeiras de uma Unidade de Tratamento Intensivo (UTI), e obtiveram um escore de 66,3. Ao contrário disso, Guessner (2006) ao avaliar equipes de saúde, encontrou um escore de 75,0, que está inserido na 'região de sucesso'. Porém além de avaliar um número muito maior de ACSs $(57,0)$, Guessner analisou profissionais de outras funções (médicos, enfermeiras, auxiliares de enfermagem, dentistas e auxiliares de consultórios de enfermagem), o que pode ter influenciado no resultado final.

O domínio psicológico foi o segundo com maior escore entre os domínios do questionário, correspondente a 58,3, que está na 'região de indefinição', assim como Paschoa, Zanei e Whitaker (2007), que encontraram uma pontuação de 60,8.

O domínio físico que neste estudo apresentou o terceiro maior escore $(55,4)$, permanecendo na 'região de indefinição', no estudo de Paschoa, Zanei e Whitaker (2007) também apresentou um escore insuficiente para a categoria $(53,1)$.

O domínio meio ambiente dos ACSs obteve 50,5 de escore, sendo o pior resultado entre os domínios, mas permanecendo na 'região de indefinição'. No estudo de Paschoa, Zanei e Whitaker (2007), este domínio também obteve o menor valor entre os 4 (49,4), assim como Guessner (2006) com 63,0 de escore. 
A avaliação do Burnout permitiu observar um quadro de estresse crônico dos ACSs, com seus respectivos níveis, revelando o envolvimento emocional nessa função. De acordo com Custódio et al. (2006), os ACSs possuem alta probabilidade de apresentar nível de estresse alterado, decorrente da baixa capacidade de adaptação aos fatores estressantes.

Os ACSs estão envolvidos diariamente em diversas situações que fazem parte de suas atividades de vida profissional, mas que contribuem significativamente para o aparecimento do estresse. Dessa forma:

\footnotetext{
Durante essas atividades lida com conflitos das famílias que atende, com entraves burocráticos que dificultam o relacionamento com a comunidade, com falta de escolaridade e condições básicas de higiene e moradia, dificuldades de entendimento com a equipe, cobranças excessivas, desvio de função, e etc (SIMÕES, 2009, p. 18).
}

De modo geral, por serem os principais meios de ligação entre a equipe de saúde e a população, a proximidade física e emocional imposta pela sua função, o contato direto com problemas dentro da equipe de saúde e nas famílias pelas quais são responsáveis se transforma em fatores de risco para o surgimento do estresse (VILELA; SILVA; JACKSON FILHO, 2010; MAIA; SILVA; MENDES, 2011).

Dentre os tipos de estratégias utilizadas pelos ACSs (fator controle, fator esquiva e fator manejo de sintomas) frente a uma situação estressante, o fator controle foi o tipo mais utilizado. O uso deste fator também foi identificado por Santos (2007), que relata que a utilização de estratégias de enfrentamento do estresse focada no fator controle, previne o surgimento do Burnout nos trabalhadores. De acordo ainda com o autor, a síndrome de Burnout está relacionada ao uso excessivo do fator esquiva (sendo o segundo fator mais utilizado).

$\mathrm{O}$ fator esquiva foi a segunda estratégia mais usada (média 3,4). $\mathrm{O}$ fator esquiva tem uma relação muito próxima com o surgimento da exaustão emocional e com o estabelecimento da síndrome de Burnout, sendo muito utilizado em ambientes estressantes como forma de se adaptar ao local de trabalho (SANTOS, 2007).

O fator manejo de sintomas foi o menos utilizado pelos ACSs. De acordo com Santos (2007), o fator manejo de sintomas, assim como o fator controle, diminui a vulnerabilidade dos ACSs ao surgimento do estresse (Burnout), de forma que uma associação de maior uso de fatores de manejo e controle em um determinado grupo de trabalhadores pode auxiliar na prevenção do surgimento do estresse (Burnout).

A correlação entre as variáveis dos questionários foi observada, mas somente entre os questionários de qualidade de vida e Coping ocupacional.

O fator esquiva apresentou correlação com o domínio psicológico. De acordo com Santos (2007), a 'fuga' frente a situações estressantes, afeta significativamente o psicológico do indivíduo, pelo surgimento de uma exaustão emocional advinda desta situação. De acordo com resultados desse estudo, o contrário também é uma resposta para a correlação.

O domínio social apresentou forte correlação com o domínio psicológico, ambos da qualidade de vida. De acordo com Vasconcellos e Costa-Val (2008), o bom relacionamento social dos ACSs com as pessoas que atende e com as que compõem a equipe de saúde, influencia diretamente na sua satisfação pessoal (domínio psicológico). A influência contrária também pode ser uma resposta para a correlação.

O domínio físico foi outro componente da qualidade de vida que se correlacionou com um domínio do próprio questionário (ambiente). De acordo com Guessner (2006), quando variáveis do domínio ambiente estão alterados (problemas financeiros, ambiente de trabalho perigoso), há riscos à saúde física do trabalhador (fadiga, vertigens, além de diversas doenças). A influência do domínio físico sobre o ambiente também pode ser uma das respostas para a correlação. 


\section{Considerações finais}

Os ACSs em sua totalidade avaliados neste estudo apresentaram idade média de aproximadamente 32 anos e um tempo na função de 68,8 meses.

O domínio social foi o que obteve o maior resultado entre os componentes da qualidade de vida dos agentes.

Em relação ao nível de Burnout, os resultados foram altos, indicando que a maioria possuía a síndrome de Burnout instalada.

As estratégias de enfrentamento do estresse indicaram que o fator mais utilizado foi o controle, o que deveria resultar em um menor nível de Burnout entre os ACSs. O resultado médio do fator esquiva foi maior que o do fator manejo de sintomas, podendo ser esta uma hipótese da instalação do Burnout nos ACSs.

As principais correlações encontradas foram entre variáveis do mesmo questionário (social e psicológico; físico e ambiente), mostrando o quanto alterado estão os aspectos da qualidade de vida dos ACSs. Porém, o resultado mais marcante é a correlação entre a utilização do fator esquiva, em situações estressantes, e o estado psicológico dos agentes, indicando uma possível resposta para a instalação do Burnout (não comprovado neste estudo).

A correlação entre a presença do estresse (Síndrome de Burnout) e alterações na qualidade de vida e na escolha de enfrentamento do estresse não foram encontrados, mas ficou clara a influência mútua das alterações da qualidade de vida em relação a tipos de enfrentamento de estresse, e entre domínios da própria qualidade de vida.

\section{REFERÊNCIAS}

BARBIRATO, C. B.; FÉLIX R.; AZEVEDO, J. C.; CORRÊA, P. L.; NÓBREGA, A. C. L.; COIMBRA, A.; VOLSCHAN, A.; MESQUITA, E. T.; DOHMANN, H. F. R.; MESQUITA, C. T. Prevalência de isquemia induzida por estresse mental. Arquivos Brasileiros de Cardiologia; v. 94, n. 3, p. 321-327, 2010. Crossef

CAMELO, S. H. H. Riscos psicossociais relacionado no trabalho das equipes de saúde da família e estratégias de gerenciamento 2006. $161 \mathrm{f}$. Tese (Doutorado em Enfermagem) Programa de Doutoramento do Interunidades, Universidade de São Paulo, Ribeirão Preto, 2006.

CUSTÓDIO, L. C.; PRATA, F. S.; SANÁBIO, G.; BRAGA, J. F.; AMARAL E SILVA, L.; MORREALE, P. G.; COSTA-VAL, R. Avaliação do estresse ocupacional em agentes comunitários de saúde da região metropolitana de Belo Horizonte - MG. Revista Brasileira de Medicina de Família e Comunidade, v. 2, n. 7, p. 1-7, out./dez. 2006.

FLECK, M. P. A; LOUZADA, S.; XAVIER, M.; CHACHAMOVICH, E.; VIEIRA, G.; SANTOS, L.; PIZON, V. Aplicação da versão em português do instrumento abreviado de avaliação da qualidade de vida 'WHOQOL-bref'. Revista de Saúde Pública, v. 34, n. 2, p. 178-183, abr. 2000. crossef

GUESSNER, C. L. S. Qualidade de vida das equipes de saúde da família do município de Timbó-SC. 2006. 94 f. Dissertação (Mestrado em Saúde e Gestão do Trabalho) - Universidade do Vale do Itajaí, Itajaí, 2006.

JIBELI, C. Questionário Jibeli para identificação preliminar da Burnout [inspirado no Maslach Burnout Inventory - MBI]. 2009. Disponível em: http://www.chafic.com.br/index_arquivos/Burnout.pdf. Acesso em: 20 set. 2011. 
LATACK, J. C. Coping with job stress: measures and future directions for scale development. Journal of Applied Psychology, v. 71, n. 3, p. 377-385, 1986. rossef

LENTINE, E. C.; SONODA, T. K.; BIAZIN, D. T. Estresse de profissionais de saúde das unidades básicas do município de Londrina. Terra e Cultura, n. 37, p. 103-123, jul./dez. 2003.

LOPES, J. Afastamento do trabalho por estresse cresce $28 \%$ em um ano no Brasil. 2011. Disponível em: <http://www.caririnoticia.com.br/2011/08/afastamento-do-trabalho-por-estressecresce-28-em-um-ano-no-brasil.html>. Acesso em: 02 Set. 2011.

LORENZ, V. R.; BENATTI, M. C. C.; SABINO, M. O. Burnout and stress among nurses in a University Tertiary Hospital. Revista Latino-Americano de Enfermagem, v. 18, n. 6, p. 10841091, nov./dez. 2010.

LOURES, D. L.; SANT'ANNA, I.; BALDOTTO, C. R. S; DE SOUSA, E. B.; DA NÓBREGA, A. C. L. Estresse mental e sistema cardiovascular. Arquivos Brasileiros de Cardiologia, v. 78, n. 5, p. 525-530, 2002. crossef

MAIA, L. D. G.; SILVA, N. D.; MENDES, P. H. C. Síndrome de Burnout em agentes comunitários de saúde: aspectos de sua formação e prática. Revista Brasileira de Saúde Ocupacional, v. 36, n. 123, p. 93-102, maio 2011.

MASLACH, C., JACKSON, S. E. The measurement of experienced Burnout. Journal of Occupational Behaviour, v. 2, n. 2, p. 99-113, 1981. Crossef

MINAYO, M. C. S.; HARTZ, Z. M. A.; BUSS, P. M. Qualidade de vida e saúde: um debate necessário. Ciência \& Saúde Coletiva, v. 5, n. 1, p. 7-18, 2000. rossef

MURTA, S. G.; TRÓCCOLI, B. T. Avaliação de intervenção em estresse ocupacional. Psicologia: Teoria e Pesquisa, v. 20, n. 1, p. 39-47, jan./abr. 2004.

PASCHOA, S.; ZANEI, S. S. V.; WHITAKER, I. Y. Qualidade de vida dos trabalhadores de enfermagem de unidades de terapia intensiva. Acta Paulista de Enfermagem, v. 20, n. 3, p. 30510, jul./set. 2007. crossef

PEDROSO, B.; PILATTI, L. A.; GUTIERREZ, G. L.; PICININ, C. T. Cálculo dos escores e estatística descritiva do WHOQOL-bref através do Microsoft Excel. Revista Brasileira de Qualidade de Vida, v. 02, n. 01, p. 31-36, jan./jun. 2010. Grossef

PINHEIRO, F. A.; TRÓCCOLI, B. T.; TAMAYO, M. R. Mensuração de Coping no ambiente ocupacional. Psicologia: Teoria e Pesquisa, v. 19, n. 2, p. 153-158, maio/ago. 2003.

ROCHA, M. C. P.; MARTINO, M. M. F. O estresse e qualidade de sono do enfermeiro nos diferentes turnos hospitalares. Revista da Escola de Enfermagem da USP, v. 44, n. 2, p. 280-286, 2010. rossef

SANTANA, M. L; CARMAGNANI, M. I. Programa saúde da família no Brasil: um enfoque sobre seus pressupostos básicos, operacionalização e vantagens. Saúde e Sociedade, v. 10, n. 1, p. 33-53, 2001. crossef 
SANTOS, T. S. Profissionais de saúde da assistência domiciliar: stress, estressores e estratégias de Coping. 2007. 101 f. Dissertação (Mestrado em Psicologia) - Pós Graduação em Psicologia, Universidade Federal do Rio de Janeiro, Rio de Janeiro, 2007.

SAUPE, R.; NIETCHE, E. A.; CESTARI, M. A.; GIORGI' M. D. M.; KRAHL M. Qualidade de vida dos acadêmicos de enfermagem. Revista Latino-Americana de Enfermagem, v. 12, n. 4, p. 636-642, jul./ago. 2004.

SEIDL, E. M. F.; ZANNON, C. M. L. C. Qualidade de vida e saúde: aspectos conceituais e metodológicos. Caderno de Saúde Pública, Rio de Janeiro, v. 20, n. 2, p. 580-588, mar./abr. 2004.

SIMÕES, A. R. O Agente comunitário de saúde na equipe de saúde da família: fatores de sobrecarga de trabalho e estresse. Revista de Saúde Pública, Florianópolis, v. 2, n. 1, p. 6-21, 2009.

TELlES, S. H.; PIMENTA, A. M. C. Síndrome de Burnout em agentes comunitários de saúde e estratégias de enfrentamento. Saúde e Sociedade, v. 18, n. 3, p. 467-478, jul./set. 2009. crossef

UMANN, J. Estresse, Coping e presenteísmo em enfermeiros hospitalares. 2011. $131 \mathrm{f}$. Dissertação (Mestrado em Enfermagem) - Programa de Pós Graduação em Enfermagem, Universidade Federal de Santa Maria, Santa Maria, 2011.

VASCONCELlOS, N. P. C.; COSTA-VAL, R. Avaliação da qualidade de vida dos agentes comunitários de saúde de Lagoa Santa - MG. Revista de Atenção Primária à Saúde, v. 11, n. 1, p. 17-28, jan./mar. 2008.

VIEIRA, I. Conceito(s) de Burnout: questões atuais da pesquisa e a contribuição da clínica. Revista Brasileira de Saúde Ocupacional, São Paulo, v. 35, n. 122, p. 269-276, jul./dez. 2010. rossef

VILELA, R. A. G.; SILVA, R. C.; JACKSON FILHO, J. M. Poder de agir e sofrimento: estudo de caso sobre agentes comunitários de saúde. Revista Brasileira de Saúde Ocupacional, São Paulo, v. 35, n. 122, p. 289-302, jul./dez. 2010. crossef 